Quark Model and High Energy Collisions 
This page is intentionally left blank 


\title{
QUARK MODEL AND HIGH ENERGY COLLISIONS
}

\author{
$\checkmark \vee$ ANISOVICH \\ $M N$ KOBRINSKY \\ J NYIRI \\ YU M SHABELSKI
}

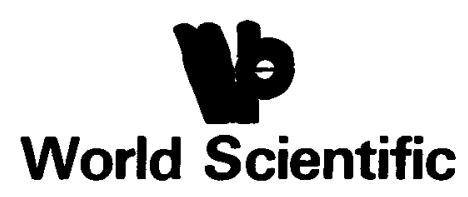


Published by

World Scientific Publishing Co Pte Ltd.

P. O. Box 128, Farrer Road, Singapore 9128

242, Cherry Street, Philadelphia PA 19106-1906, USA

\section{Library of Congress Cataloging in Publication Data \\ Main entry under title:}

Quark model and high energy collisions.

1. Quarks. 2. Nuclear reactions. 1. Anisovich, V. V. II. Kobrinsky M. N. III. Nyiri J. IV. Shabelski Yu. M. $\begin{array}{llll}\text { QC793.5.Q252Q364 } & 1985 & 539.7 ' 21 & 85-20350\end{array}$

ISBN $9971-966-68-9$

Copyright $\odot 1985$ by World Scientific Publishing Co Pte Ltd.

All rights reserved. This book, or parts thereof, may not be reproduced in any form or by any means, electronic or mechanical, including photocopying, recording or any information storage and retrieval system now known or to be invented, without written permission from the Publisher.

Printed in Singapore by Singapore National Printers (Pte) Ltd. 


$$
\text { To the memory of }
$$

\section{Vladimir Mikhailovitch Shekhter}


This page is intentionally left blank 


\section{PREFACE}

This book treats one of the directions in elementary particle physics, namely, the application of the quark model to high energy hadron interactions. The main topics under consideration are binary processes, multiparticle production and hadron-nucleus collisions. Since we are considering a relatively restricted subject matter, we have the possibility of presenting a detailed exposition of the results as well as the required calculational techniques. We have made an effort to elucidate the material to such an extent that a novice in the field should be able to make his own calculations after the book is studied. The audience for whom the book is written is assumed to be familiar with quantum field theory, including Feynman diagrams, and also to be acquainted with the eperimental situation in high energy physics.

The subjects considered are inevitably connected with some other domains of elementary particle physics. In our effort to make our book as self-contained as possible we have discussed the necessary background material from these adjacent domains. Chapter 2 gives a concise account of the main experimental results in high energy strong interactions; the foundations of the Regge-pole phenomenology and multiperipheral dynamics are also briefly described there. In Chapter 3 the methods for treating composite systems are explained, while Chapter 4 discusses the theory of multiple rescattering at high energies. The present status of the quark model is reviewed in Chapter 5 . 
The material covered in Chapters 2 and 5 are described in more detail in books like

J.C. Polkinghorne, Models of High Energy Physics, Cambridge Univ. Press, Cambridge (1975).

P.D.B. Collins, An Introduction to Regge Theory and High Energy Physics, Cambridge Univ. Press, Cambridge (1975).

K. Huang, Quarks, Leptons \& Gauge Fields, World Scientific, Singapore (1982).

F.E. Close, An Introduction to Quarks and Partons, Academic Press, New York (1979).

Some auxiliary material has been relegated to the Appendices and could probably aid the reader in making various explicit calculations.

We would like to thank A.A. Anselm, I.M. Dremin, S.S. Gerstein, V.G. Grishin, E.M. Levin, N.N. Nikolaev, M.G. Ryskin, E.V. Shuryak for extremely helpful discussions of the problems considered in the book. We are also deeply indebted to V.N. Gribov: his comments were extremely helpful for us. We are happy to thank our friends and colleagues V.M. Braun, L.G. Dakhno, A.K. Likhoded and P.E. Volkovitski: some papers written in collaboration with them are reflected in this work. The authors are grateful to K.K. Phua for his encouragement in writing this book. Two of us (V.V.A. and Yu.M.S.) are much obliged to $0 . I$. Sumbaev for the permanent support of the investigations presented here: the book could hardly be accomplished without it.

We are specially indebted to our deceased friend V.M. Shekhter. Our collaboration with him has provided much material which is being passed on in this book. This book is dedicated to his memory.

Gatchina, USSR

Moscow, USSR

Budapest, Hungary
V.V. Anisovich

M.N. Kobrinsky

J. Nyiri

Yu.M. Shabelski 


\section{CONTENTS}

Preface vii

CHAPTER 1: INTRODUCTION 1

1.1 The Quark-Gluon Structure of Hadrons 1

1.2 Dressed Quarks 6

$\begin{array}{lll}\text { CHAPTER 2: HIGH ENERGY HADRON INTERACTIONS } & 18\end{array}$

2.1 General Picture of the Energy
Dependence for Hadron Interactions

2.2 Multiparticle Production and Inclusive Processes 23

2.2.1 Average multiplicity and limited transverse
momenta

2.2.2 Kinematic variables for the secondary particles 26

2.2.3 Secondary particle distributions in the central and the fragmentational regions 29 2.2.4 Multiperipheral collisions 32

2.3 Regge-Pole Phenomenology 34

2.3.1 Binary reactions 34

2.3.2 Inclusive reactions 46

2.3.3 The AGK cutting rules $\quad 49$

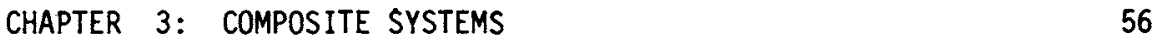

3.1 Non-Relativistic Composite Systems 56

Lorentz-Invariant Description of
Non-Relativistic Systems

3.3 The Inclusion of Relativistic Effects
in Composite Systems 
4.1 Scattering on a Two-Body System -

Glauber Approximation
4.2 Scattering on a Two-Body System Inelastic Screening

4.3 Scattering on N-Body Systems. General Formulas 105

4.4 Nuclear Target Scattering $(A \geq 10)$

4.5 Diffraction Excitation of the Nuclei and Noncoherent Scattering

4.6 Inelastic Screening in Nuclear Target Scattering and the Method of Eigenstates

4.7 Calculation of the Noncoherent Process Cross Sections through Diagram-Cutting Rules

CHAPTER 5: QUARK MODEL AND STATIC FEATURES OF HADRONS

5.1 Hadron Zoology 129

5.1.1 Mesons 130

5.1.2 Baryons 136

5.2 Magnetic Moments of Baryons and Radiative
Decays of Vector Mesons

5.3 Hadron Masses and Potential Models 143

5.4 Short-Range Interaction in the Quark Model 149

$\begin{array}{lll}\text { CHAPTER 6: BINARY PROCESSES IN THE QUARK MODEL } & 161\end{array}$

6.1 Confinement and Impulse Approximation 161

6.2 Elastic Scattering of Quarks 172

6.2.1 Total cross sections 173

6.2.2 Elastic cross sections at small momentum
transferred

6.2.3 The estimates of the dressed quark radius 181

6.3 Flavour-Exchange Reactions 182

6.3.1 Cross section 182

6.3.2 Polarization phenomena 190

6.4 Regge-Pole Exchanges in the Quark Model 199

6.5 Multiple Quark Scatterings 205

6.6 Hadron Diffraction-Dis sociation Processes 210

CHAPTER 7: MULTIPARTICLE PRODUCTION IN THE QUARK MODEL 220

7.1 Space-Time Picture and Spectator Mechanism 220

7.2 Secondary Particles and Their Momentum
Distributions

7.3 Quark Statistics. Hadron Content of the
Secondaries

7.3.1 Statistical rules 233

7.3.2 Hadron ensembles 239

7.3.3 Meson-baryon ratios 249 
7.4 Total and Partial Multiplicities 256

7.5 Inclusive Spectra 268

7.6 Multiple Rescattering and Scale Breaking
at Superhigh Energies

7.6.1 Shadow corrections 283

7.6.2 Scale breaking 286

CHAPTER 8: HADRON-NUCLEUS COLLISIONS 293

8.1 Spectator Mechanism and Its Consequences 294

8.1.1 A-Dependence of the inclusive spectra
in the fragmentation region

8.1.2 Multiplicity ratio of $\mathrm{pA}$ and $\pi \mathrm{A}$ collisions
in the central region of the spectrum

8.2 Inclusive Spectra in Hadron-Nucleus Collisions 307

8.3 Inelastic Diffraction Scattering of Hadrons on Nuclei 312

8.4 Scale Breaking in Hadron-Nucleus Interactions 321

CHAPTER 9: RECENT MODEL APPROACHES TO MULTIPLE PRODUCTION PHENOMENA

325

9.1 The Scheme of Dual Topological Unitarization (DTU)

9.2 The Recombination Model 342

9.3 The Lund Fragmentation Model 348

APPENDIX A: NOTATIONS, NORMALIZATION CONDITIONS AND ALL THAT 360

$\begin{array}{lll}\text { A.1 Metric } & 360\end{array}$

A.2 SU(N) Groups 360

A.3 Y Matrices and 4-Component Spinors 363

A.4 Amplitude Normalization Conditions 365

APPENDIX B: CROSS SECTIONS AND AMPLITUDE DISCONTINUITIES 366

B.1 Exclusive and Inclusive Cross Sections 366

B.2 Amplitude Discontinuities and Unitary
Condition

APPENDIX C: HADRON WAVE FUNCTIONS 374

C.1 S-wave Mesons 374

C.2 P-wave Mesons $\quad 375$

C. 3 S-wave Baryons $\quad 379$

$\begin{array}{ll}\text { C.4 P-wave Baryons } & 379\end{array}$

C.4.1 Decouplet states $\quad 380$

C. 4.2 Octet states 383

C.4.3 Singlet states 397

C.4.4 Mixing angles and decay schemes 399 
APPENDIX D: THE HADRON CONTENT OF MESON AND BARYON ENSEMBLES

APPENDIX E: SPECTRA OF THE RESONANCE DECAY PRODUCTS 